\title{
Neue kantonale Koordinationsstelle Weiterbildung Hausarztmedizin am BIHAM
}

Im Rahmen der anstehenden grösseren Veränderungen am BIHAM (Lehrstuhl für Hausarztmedizin, Umzug in neue Räumlichkeiten) durfte ich am 01.08.2013 die neu geschaffene und vom Kanton Bern finanzierte Stelle der Koordinatorin Weiterbildung Hausarztmedizin antreten. Nach jahrelanger Tätigkeit in einer Hausarztpraxis mit meinem Partner bin ich gespannt auf die neue Herausforderung. An Arbeit wird es mir nicht mangeln, obwohl Frau Dr. R. Ahrens in den vergangenen Jahren bereits bedeutende und wertvolle Vorarbeit geleistet hat.

Zu meinen wichtigsten Aufgaben gehören:

- die persönliche Beratung und Begleitung von Assistenzärzten in Weiterbildung Hausarztmedizin und von Wiedereinsteigern im Rahmen der Laufbahn- und Karriereplanung;

- die Vermittlung von Praxisassistenzen;

- die Verwaltung und Evaluation der aktuell 7 Rotationsstellen in Chirurgie, Orthopädie, ORL, Pädiatrie, Radiologie, Psychiatrie und Viszeralchirurgie sowie die Schaffung neuer Rotationsstellen;

- der Aufbau eines hausarztspezifischen Curriculums;

- die Organisation und Durchführung von praxisnahen Kursen für Assistenzärzte in Weiterbildung Hausarztmedizin;

- die Kontaktpflege mit den Klinikdirektoren;

- die Information der interessierten Assistenzärzte über das Angebot der Koordinationsstelle, inkl. Stellenbörse;

- die Unterstützung der Hausärzte bei der Suche nach Praxisnachfolgern.

Mit diesem Angebot und in Zusammenarbeit mit Ihnen und den vielen Partnern hoffen wir, die Attraktivität der Hausarztmedizin bei den Assistenzärzten zu erhöhen und trotz einer breiten und qualitativ hochstehenden Weiterbildung die Weiterbildungszeit möglichst kurz zu halten.

Und zu guter Letzt: Durch den Ausbau der Weiterbildungsangebote soll der hausärztliche Nachwuchs für die Zukunft sichergestellt werden.

Ihre Anfragen und Anregungen freuen mich und ich werde sie in Zukunft gerne aufnehmen.

Zugunsten einer besseren Lesbarkeit wird in diesem Artikel nur die männliche Form verwendet.

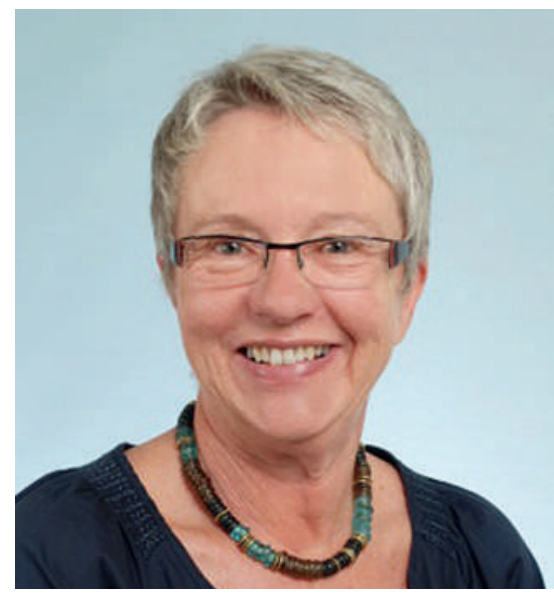

Provisorisches Programm Weiterbildungskurse 2014:

- Weichteil-Rheumatismus und Infiltrationen

- Kinder-Notfälle in der Hausarztpraxis

- EKG-Quiz für den Hausarzt

- Polypharmazie

- Motivierende Gesprächsführung: Training mit Schauspielpatienten

- Immobilisationstechnik in der Praxis

Weitere Informationen siehe www.biham.unibe.ch/ Weiterbildung.

\section{Korrespondenz:}

Dr. med. Beatrice Molinari

Koordinatorin Weiterbildung

Universität Bern

Berner Institut für Hausarztmedizin

Gesellschaftsstrasse 49

3012 Bern

beatrice.molinari[at]biham.unibe.ch 